\title{
Construction and Qualification of the Pre-Series MQM Superconducting Quadrupoles for the LHC Insertions
}

\author{
R. Ostojic, N. Catalan Lasheras, J. Lucas, W. Venturini Delsolaro, and D. Landgrebe
}

\begin{abstract}
The LHC insertions will be equipped with individually powered MQM superconducting quadrupoles, produced in three versions with magnetic lengths of $2.4 \mathrm{~m}, 3.4 \mathrm{~m}$, and $4.8 \mathrm{~m}$. The quadrupoles feature a $56 \mathrm{~mm}$ aperture coil, designed on the basis of an $8.8 \mathrm{~mm}$ wide Rutherford-type NbTi cable for a nominal gradient of $200 \mathrm{~T} / \mathrm{m}$ at $1.9 \mathrm{~K}$ and $5390 \mathrm{~A}$. A total of 96 quadrupoles are in production in Tesla Engineering, UK. In this report we describe the construction of the pre-series MQM quadrupoles and present the results of the qualification tests.
\end{abstract}

Index Terms-Insertions, LHC, quadrupoles, superconducting.

\section{INTRODUCTION}

$\mathbf{T}$ HE optics flexibility of the LHC insertions is provided by the individually powered quadrupoles in the dispersion suppressors and matching sections. A special superconducting quadrupole has been developed for these sections of the collider, featuring a $56 \mathrm{~mm}$ aperture coil based on an $8.8 \mathrm{~mm}$ wide cable and designed for a nominal gradient of $200 \mathrm{~T} / \mathrm{m}$ at $5390 \mathrm{~A}$ [1]. The magnets are assembled in several different types of quadrupole cold masses with lengths of up to $11.3 \mathrm{~m}$, comprising several magnets powered in series [2].

The MQM quadrupole, Fig. 1, consists of two identical, independently powered apertures, which are assembled in a two-in-one yoke structure. Three versions of the MQM quadrupole are required for the LHC, with magnetic lengths of $2.4 \mathrm{~m}, 3.4 \mathrm{~m}$ and $4.8 \mathrm{~m}$. The main parameters of the quadrupole are given in Table I. The MQM design was validated on several single and twin-aperture model magnets [3]. Following the development work at CERN, the contract for the supply of 96 MQM quadrupoles was adjudicated to Tesla Engineering (UK).

In this paper we present the initial experience in the fabrication of the pre-series MQM quadrupoles, and present the results of the qualification tests.

\section{MAGNET FABRICATION}

For the production of the MQM quadrupoles, Tesla Engineering has set-up a new production unit in Lancing, Sussex. All the required heavy tooling for coil fabrication and magnet

Manuscript received October 20, 2003.

R. Ostojic, N. Catalan Lasheras, and W. Venturini Delsolaro are with CERN, Accelerator Technology Division, 1211 Geneva 23, Switzerland (e-mail: Ranko.Ostojic@cern.ch).

J. Lucas was with CERN, Geneva. He is presently with ELYTT Energy, Portugalete, Spain.

D. Landgrebe is with the Tesla Engineering, Storrington, UK.

Digital Object Identifier 10.1109/TASC.2004.829046

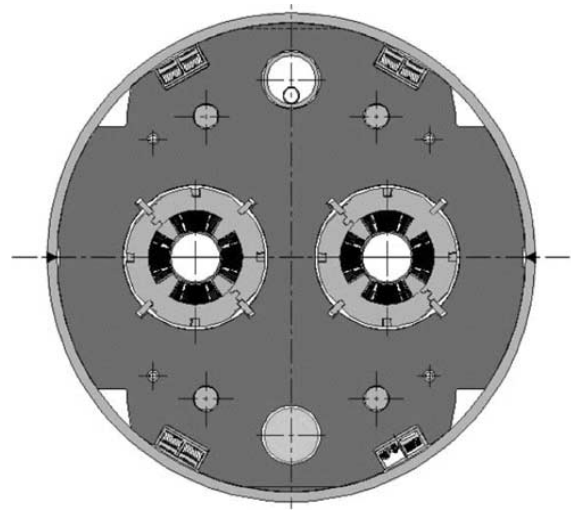

Fig. 1. Cross-section of the MQM insertion quadrupole.

TABLE I

MAIN PARAMETERS OF THE MQM QUADRUPOLE

\begin{tabular}{lc}
\hline \hline Coil inner diameter & $56 \mathrm{~mm}$ \\
Magnetic length & $2.4 \mathrm{~m}, 3.4 \mathrm{~m}, 4.8 \mathrm{~m}$ \\
Operating temperature & $1.9 \mathrm{~K}$ \\
Nominal gradient & $200 \mathrm{~T} / \mathrm{m}$ \\
Nominal current & $5390 \mathrm{~A}$ \\
Peak field in coil & $6.13 \mathrm{~T}$ \\
Quench field & $7.58 \mathrm{~T}$ \\
Stored energy per aperture & $64.3 \mathrm{~kJ} / \mathrm{m}$ \\
Inductance per aperture & $4.44 \mathrm{mH} / \mathrm{m}$ \\
Cable parameters & \\
Width & $8.8 \mathrm{~mm}$ \\
Mid-thickness & $0.845 \mathrm{~mm}$ \\
Keystone angle & $0.91 \mathrm{deg}$. \\
No of strands & 36 \\
Strand dia. & $0.475 \mathrm{~mm}$ \\
Cu/SC Ratio & 1.75 \\
Filament dia. & $6 \mu \mathrm{m}$ \\
$\mathrm{j}_{\mathrm{c}}\left(\mathrm{A} / \mathrm{mm}^{2}, 4.2 \mathrm{~K}\right.$ and $\left.5 \mathrm{~T}\right)$ & 2800 \\
\hline
\end{tabular}

assembly was installed in this facility. A $6 \mathrm{~m}$ long winding machine of the racetrack type and a $5 \mathrm{~m}$ curing press were installed for winding and curing of all coil types. The commissioning of the tooling was performed on several trial coils. The running-in of the dedicated collaring press and of the assembly procedures was done on short magnet assemblies.

Particular attention during the production of the pre-series coils was paid to refining of the coil winding technique and of the parameters of the curing press. The satisfactory quality of turn bonding in the straight section and coil ends, shown in Fig. 2, was achieved early in the process, and only minor corrections of the shape of the end spacers were necessary. However, several iterations were required to achieve the correct azimuthal 


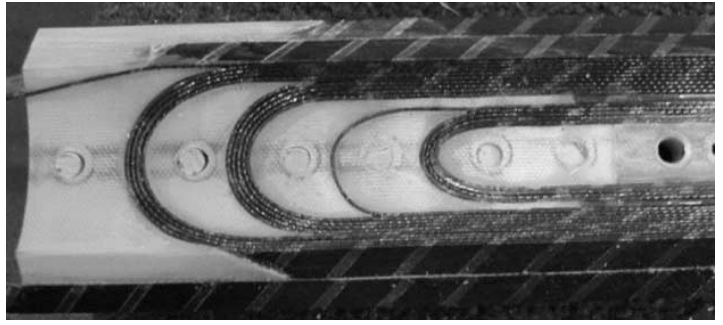

Fig. 2. View of the lead end of the cured MQM coil, showing the good quality of turn bonding and fit to the end spacers.

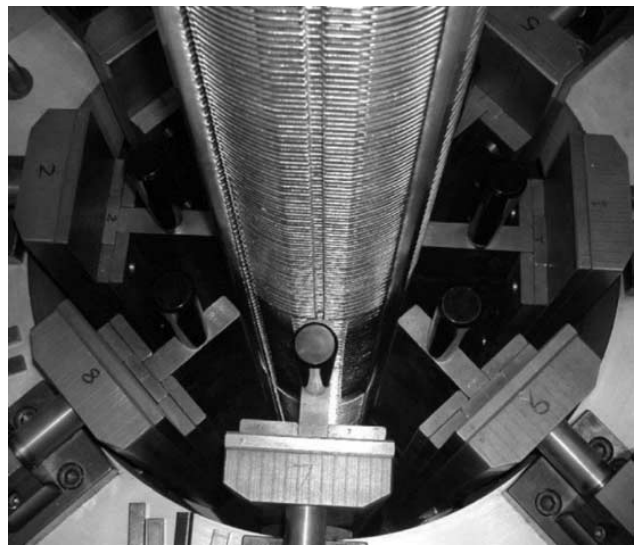

Fig. 3. Collaring of the quadrupole aperture in the eight-way collaring press. The full-length collaring keys are pressed in steps of $200 \mathrm{~mm}$ in two passes.

size of the coils. Measurements performed on 22 coils produced in the initial production phase showed that the coil sizes are within $\pm 25 \mu \mathrm{m}$ of the nominal value, which corresponds to a pre-stress of $70 \pm 15 \mathrm{MPa}$. This level of reproducibility was not achieved for the coil ends, which is partly due to the deflection of the curing press in different curing configurations. For this reason, all coil ends were individually shimmed.

The assembly of a quadrupole aperture involves several delicate operations, in particular the assembly of the quench heaters and of the ground insulation. The horizontal assembly bench designed for this purpose proved versatile and the collaring mandrel precise in locating the coils. Collaring of the aperture is done in a vertically standing press, shown in Fig. 3. The assembly is initially compressed starting from the middle of the assembly toward the ends to align the collars and remove all slack. The full-length collaring keys are thereafter inserted in two passes. The collared assembly is completed with end plates, which are welded to the key extremities, Fig. 4. The plates provide longitudinal support to the coils and serve for locating the connection box.

The final stage of magnet fabrication is the assembly of yoke laminations around the collared apertures. A yoking tower, shown in Fig. 5, was built for this operation, providing an efficient means for stacking and longitudinal compression of the laminations.

The construction of the pre-series magnets revealed several areas for improvement of the winding technique and of the assembly procedures. While the production rate of one coil a day was satisfactory for the pre-series phase, it needs to be improved to meet the project goals. In addition, adherence to strict quality

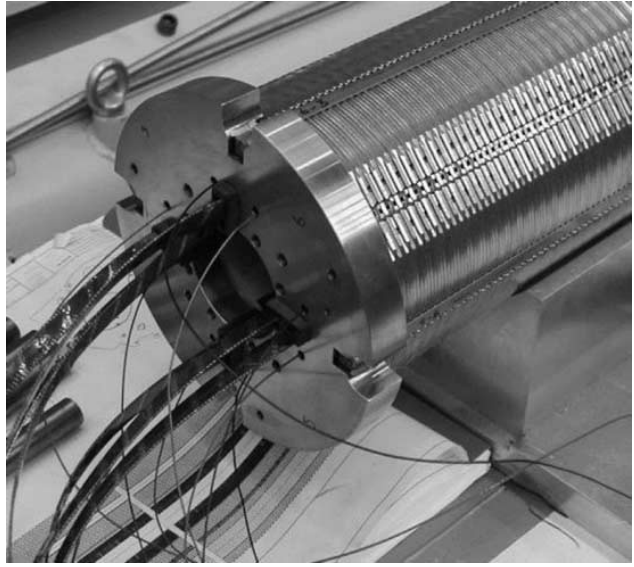

Fig. 4. The lead end of the completed collared aperture, with the coil leads ready for jointing in the connection box. The instrumentation and quench heater wires are also routed through the connection box.

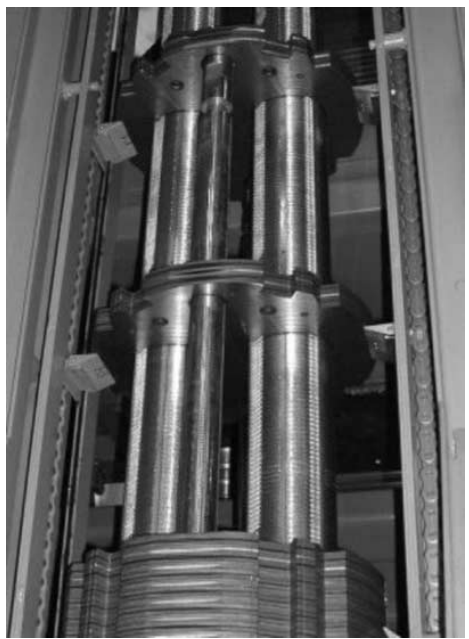

Fig. 5. Completion of the MQM quadrupole in the vertical yoking tower.

control rules, in particular the electrical tests, was found to be essential for maintaining the quality of production.

\section{QUALIFICATION TESTS}

Four pre-series MQM quadrupoles were tested in the $1.9 \mathrm{~K}$ vertical test facility at CERN, two MQMC $(2.4 \mathrm{~m})$ and two MQM magnets $(3.4 \mathrm{~m})$. The MQML magnets $(4.8 \mathrm{~m})$ could not be tested in this facility due to the limited height of the cryostat, and will be qualified as part of the completed cryo-magnets in the horizontal test station at CERN.

\section{A. Power Tests}

The main goal of the powering tests was to determine the quench training behavior of the magnets in superfluid and helium at $4.5 \mathrm{~K}$, and to confirm the effectiveness of the quench protection system. In addition, the electrical integrity of the magnets was checked in all phases of magnet testing.

The quench training of the four MQM-type quadrupoles is shown in Fig. 6. The magnets were powered to the nominal current of $5390 \mathrm{~A}$, corresponding to the operational gradient of $200 \mathrm{~T} / \mathrm{m}$ in the LHC, and training was expected to occur around this current. In fact, the first three magnets (MQMC-01, 


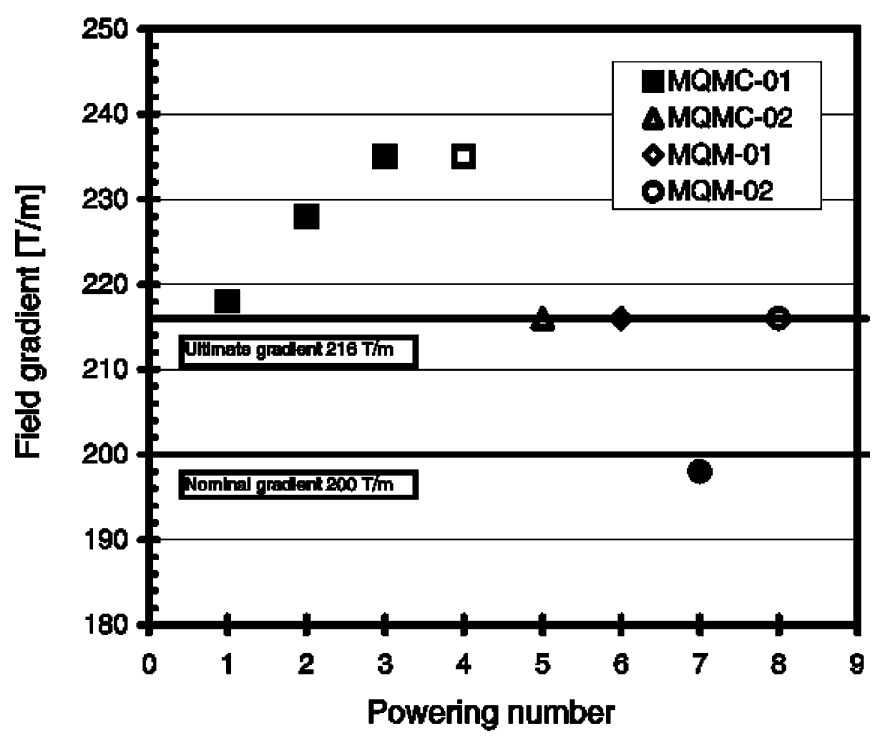

Fig. 6. Quench training of the four pre-series MQM quadrupoles at $1.9 \mathrm{~K}$. Open symbols correspond to current ramp-up without quench.

MQMC-02 and MQM-01) did not quench on first powering up to the ultimate current in the LHC of 5820 A. MQMC-01, which was trained above this current, reached the conductor limit in three quenches. Its training state was confirmed after a thermal cycle, when the magnet was powered without quench to $6300 \mathrm{~A}$. MQM-02 had a first training quench at $5263 \mathrm{~A}$ (198 T/m), slightly below the nominal current, but went up to the ultimate limit on the next ramp-up without further training. At $4.5 \mathrm{~K}$, all magnets reached the conductor limit without quench. The very successful training results of the pre-series magnets confirmed the robustness of the design and the quality of the production technique, but also indicated that high level of production quality must be maintained to assure successful performance.

MQM magnets are protected by quench heaters mounted on the outer surface of the coils, and arranged in two independent circuits. Due to the high current density in the copper fraction of the cable during quench, the quench heaters must be fired promptly and must react rapidly. The quench heater delays were systematically measured on all four pre-series quadrupoles over the full range of operating currents. The results, shown in Fig. 7, indicate that the quench heaters react within $10-15 \mathrm{~ms}$ at nominal current, and within $60 \mathrm{~ms}$ at low currents. Furthermore, the measured data are remarkably consistent between different magnet types and follow closely the exponential law determined for prototype magnets [3].

\section{B. Field Measurements}

The magnetic field of the quadrupoles was measured with two separate systems: a system based on a $750 \mathrm{~mm}$ rotating coil designed for warm measurements in the factory, and a system comprising five $220 \mathrm{~mm}$ coils adjacently mounted on a $1.2 \mathrm{~m}$ rotating shaft for measurements in the vertical test facility at CERN. The first system was used to obtain local and integral field data after quadrupole collaring and yoking, and is intended for early identification of assembly errors. The system used for cold measurements cannot give integral data. However, the shaft

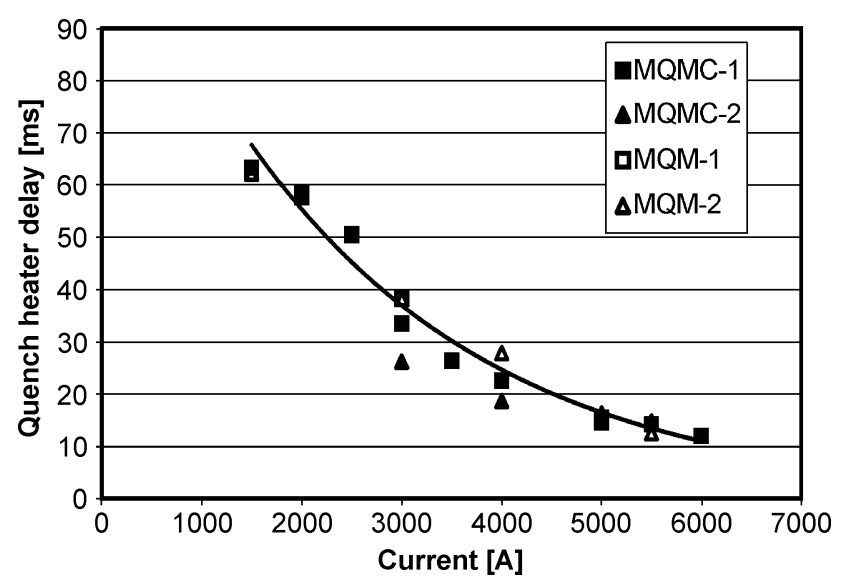

Fig. 7. Quench heater delays measured in the four pre-series MQM quadrupoles.

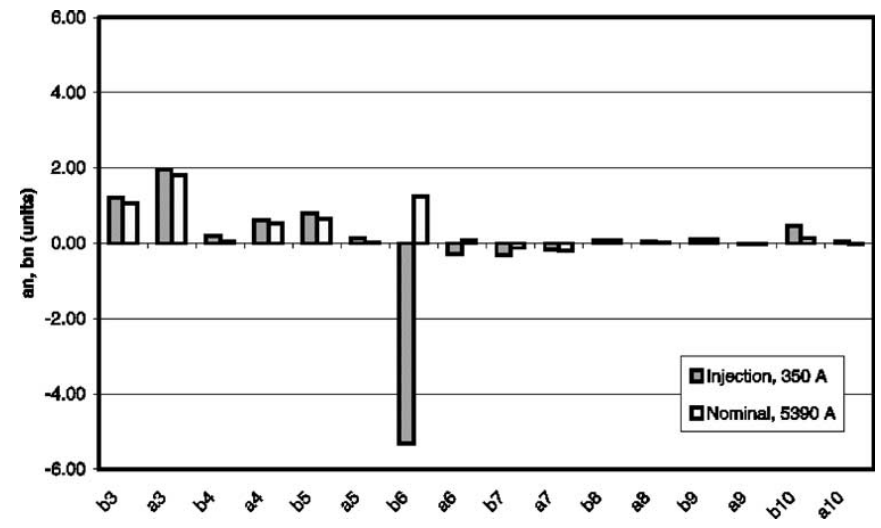

Fig. 8. Field multipoles of MQMC-01, measured at injection (350 A) and nominal $(5390 \mathrm{~A})$ currents. The multipoles are given in units of $10^{-4}$ of the quadrupole field at the reference radius of $17 \mathrm{~mm}$.

is positioned so as to cover the magnet end and part of the straight section.

The average transfer function in the straight section of the quadrupoles at room temperature is $34.53 \mathrm{~T} / \mathrm{m} / \mathrm{kA}$ for individual collared apertures and $36.83 \mathrm{~T} / \mathrm{m} / \mathrm{kA}$ for completed magnets. At $1.9 \mathrm{~K}$, the transfer function is $37.53 \mathrm{~T} / \mathrm{m} / \mathrm{kA}$ at $2500 \mathrm{~A}$, and remains constant up to $5800 \mathrm{~A}$. The spread of the magnetic lengths, deduced from the warm integral measurements, is of the order of $1 \mathrm{~mm}$.

The field harmonics for the magnet MQMC-01, typical of the series, are shown in Fig. 8. As for the transfer function, the field harmonics show very little variation above $2000 \mathrm{~A}$. Below $2000 \mathrm{~A}$, the persistent currents give rise to hysteresis, as shown in Fig. 9 for the $b_{6}$ multipole. The design of the coil is such that the geometric $b_{6}$ of 1.5 units partially compensates the $b_{6}$ at injection, which is -5.8 units at $350 \mathrm{~A}$ on the up-ramp. The $\mathrm{b}_{6}$ decays by 0.3 units after $1000 \mathrm{~s}$ at the injection plateau of $350 \mathrm{~A}$.

As a general rule, the allowed harmonics are very similar in the four pre-series magnets, both in integral values and longitudinal distributions. This is not the case for the nonallowed harmonics of the low order. For example, the $c_{3}$ field component $\left(c_{3}=\sqrt{ }\left(a_{3}^{2}+b_{3}^{2}\right)\right)$ varies in absolute value in the range from 0.5 units to 3 units. Although within the specifications, such 


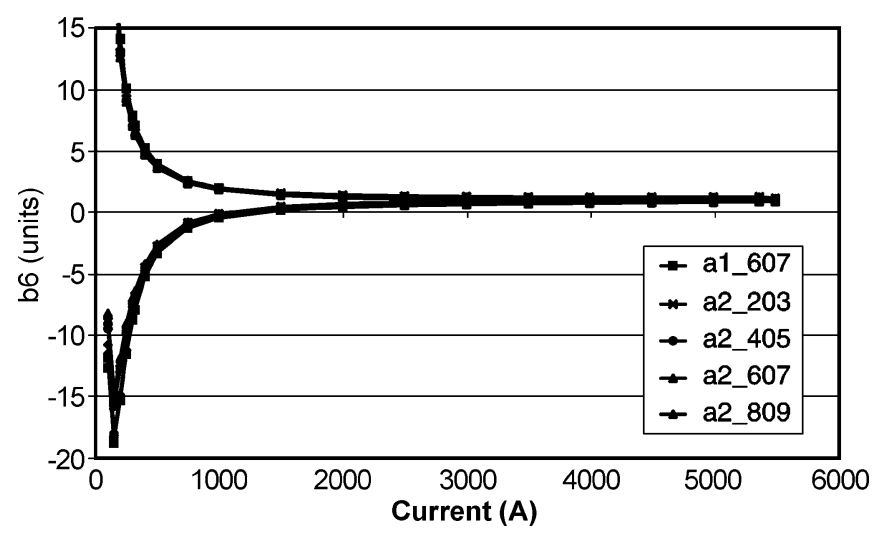

Fig. 9. Hysteresis of the $b_{6}$ multipole in the two apertures of MQMC-01. The hysteresis width and the geometric values, given at several positions in aperture 2 , are homogeneous along the magnet length.

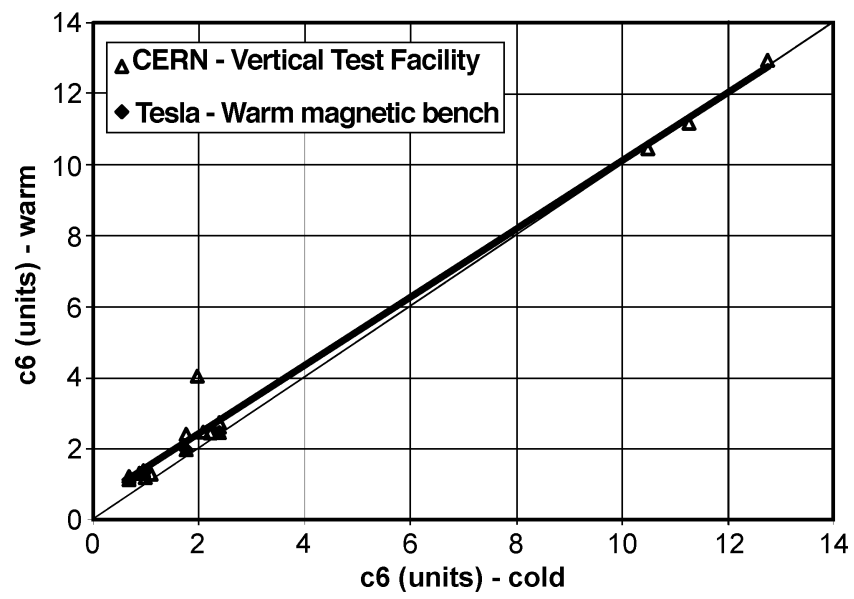

Fig. 10. Warm-cold correlation for the $c_{6}$ field component in the straight section (in the range of 1 to 2 units) and magnet ends (in the range of 10 to 12 units) of the four pre-series MQM quadrupoles. Data from factory measurements (closed symbols), corresponding to the same location as the straight section cold data, are also included.

a range indicates that the coil production and assembly procedures are not yet fully controlled, which is partly the result of the training of the production team and running-in of the tooling.

An important result of the qualification tests is that the measurements at $1.9 \mathrm{~K}$ could be correlated to those made during magnet fabrication. In Fig. 10, the $c_{6}$ measurements performed at $1.9 \mathrm{~K}$ are compared with those after magnet warm-up. Apart from the straight sections of the magnets, data for field multipoles in the ends are also included, which extend the range of correlation up to 12 units. In the same figure, the measurements performed in the factory are also shown. The available data show a high degree of correlation and a shift in $c_{6}$ of about 0.5 units on cool-down. Furthermore, the two sets of warm measurements also correlate well. As a result, the quadrupole field quality can be predicted from the fabrication data with a high level of confidence, several months ahead of cold tests. If necessary, corrective actions can be made effectively with least delay.

\section{CONCLUSION}

As part of the industrialization program of the MQM superconducting quadrupoles, a pre-series of four magnets was built by Tesla Engineering, UK. The magnets were tested in the $1.9 \mathrm{~K}$ vertical test facility at CERN. All magnets achieved the ultimate operating gradient in the LHC of $216 \mathrm{~T} / \mathrm{m}$, three without any and one magnet with a single training quench. The field quality of the magnets is within specification. However, the nonallowed harmonics of the low order indicate that the azimuthal size of the coils should be closely monitored. The very encouraging results of the pre-series production confirmed the robustness of the design and the quality of the production techniques. They also indicated that quality of production must be maintained to assure successful performance of the series magnets.

\section{ACKNOWLEDGMENT}

The authors would like to thank the technicians in Tesla Engineering for their effort in technology transfer, and the team of the LHC vertical test facility for their enthusiasm in testing these magnets.

\section{REFERENCES}

[1] R. Ostojic, "The LHC insertion magnets," IEEE Trans. Appl. Supercond., vol. 12, no. 1, pp. 196-201, March 2002.

[2] R. Ostojic, J. Lucas, G. Kirby, J. C. Perez, and H. Prin, Design, Assembly and Mechanical Behavior of the Pre-Series LHC Insertion Quadrupoles.

[3] J. Lucas, R. Ostojic, D. Tommasini, W. Venturini-Delsolaro, and D. Landgrebe, "Performance of the final prototype of the 6-kA matching quadrupoles for the LHC insertions and status of the industrialization program," IEEE Trans. Appl. Supercond., vol. 13, no. 2, pp. 1309-1312, June 2003. 